http://dx.doi.org/10.5935/1981-2965.20140035

Artigo Cientifico

http://www.higieneanimal.ufc.br

Parasitismo por Phthiraptera em Gallus gallus (Linnaeus, 1758) da raça Brahma

Gabriela Hemylin Ferreira Moura ${ }^{1}$, Mikael Almeida Lima ${ }^{2}$, Josivania Soares Pereira ${ }^{3}$, Ivana Cristina Nunes Gadelha ${ }^{4}$, Zuliete Aliona Araújo de Souza Fonseca ${ }^{5}$, Marcelle Santana de Araujo ${ }^{6}$, Sílvia Maria Mendes Ahid ${ }^{7}$

Resumo: A infestação por ectoparasitos em aves causa irritações locais, diminuição da postura, perda de peso, anemia e óbito.Objetivou-se relatar a infestação por Phthiraptera mastigadores Amblycera e Ischnocera em aves da espécie Gallus gallus, da raça Brahma, com variações de coloração Dark (preto) e Light (branco) em Pendências, Rio Grande do Norte, Brasil. Aves G. gallus da raça Brahma, criadas em sistema extensivo, foram inspecionadas para busca de ectoparasitos, uma vez que apresentava sinais de infestação, dentre eles inquietação e perda de penas. Após contenção mecânica dos animais, coletou-se Phthiraptera nas áreas corpóreas da cabeça, pescoço, asas, dorso e ventre. Os ectoparasitos recuperados foram armazenados em frascos com álcool a $70^{\circ}$ e enviados ao Laboratório de Parasitologia Animal da Universidade Federal Rural do Semi-Árido. Posteriormente, foram clareados em solução de hidróxido de potássio a $10 \%$ e montados entre lâmina e lamínula. A identificação foi feita utilizando-se chaves taxonômicas, auxiliada por microscópia óptica de luz. Os ectoparasitos foram observados em diferentes estágios de vida. Observou-se que $40 \%$ dos animais apresentaram infestação. Recuperou-se 220 Phthiraptera identificados como: 80 Lipeurus caponis; 92 Menopon gallinae e 48 Goniocotes sp. Registra-se a infestação por $L$. caponis, M. gallinae e Goniocotes sp. em G. gallus da raça Brahma, em Pendências, Rio Grande do Norte.

Palavras-chave: Amblycera, Ischnocera, Pediculose, Piolho 


\section{Parasitism by Phthiraptera in Gallus gallus (Linnaeus, 1758) Breeds Brahma dark and Brahma light}

Abstract: The infestation by ectoparasites in birds causes local irritation, impaired posture, weight loss, anemia and óbito. Objetivou-reporting Phthiraptera infestation and chewing Amblycera Ischnocera in poultry of Gallus gallus species, the Brahma breed, with color variations Dark (black) and light (white) in Pendências, Rio Grande do Norte, Brazil. Birds G. gallus Brahma breed, raised in extensive system were inspected to search for ectoparasites, once showed signs of infestation, including restlessness and loss of feathers. After mechanical restraint of the animals was collected in Phthiraptera tangible areas of the head, neck, wings, back and belly. Ectoparasites recovered were stored in vials with alcohol at $70^{\circ}$ and sent to the Laboratory of Animal Parasitology of the Universidade Federal Rural do Semi-Árido. Later, they were cleared in a solution of potassium hydroxide $10 \%$ and mounted between a slide and cover slip. Identification was made using taxonomic keys, aided by optical light microscopy. Ectoparasites were observed at different stages of life. It was observed that $40 \%$ of the animals showed infestation. Recovered 220 Phthiraptera identified as: 80 Lipeurus caponis; 92 Menopon gallinae e 48 Goniocotes sp. Join infestation L. caponis, M. gallinae and Goniocotes sp. in G. gallus Brahma breed in Pendências, Rio Grande do Norte.

Keywords: Amblycera, Ischnocera, louse, lice

\footnotetext{
${ }^{1}$ Discente do curso de graduação em Medicina Veterinária da Universidade Federal Rural do Semi-Árido (UFERSA). gabi.hemylin@ hotmail.com

${ }^{2}$ Discente do curso de graduação em Medicina Veterinária da UFERSA. mikalima@live.com

${ }^{3}$ Bióloga, técnica do Laboratório de Parasitologia Animal e doutoranda do Programa de PósGraduação em Ciência Animal da UFERSA. josigej@ufersa.edu.br

${ }^{4,5}$ Médicas veterinárias e doutorandas do Programa de Pós-Graduação em Ciência Animal da UFERSA. ivanacris@bol.com.braalionahta@hotmail.com

${ }^{6,7}$ Docentes do Departamento de Ciência Animal da UFERSA.

Autor para correspondência. E.Mail: * ahid@ufersa.edu.br

Recebido em 10/03/2014. Aceito em 14/05/2014
} 


\section{Introdução}

As aves da raça Brahma possuem origem asiática. São derivadas de um cruzamento do Malaio com o Cochin que através de um aprimoramento genético feito pelos americanos, resultaram à Brahma Light, Brahma Dark e Brahma Buff (Figueiredo et al., 2003). Possuem como características: aspecto elegante, demasiada beleza, cores fortes, plumagem macia ajustada ao corpo, coloração da pele amarela, cor da casca dos ovos marromclaro, bico curvado e, como diferencial, a presença de penas nos membros posteriores até o dedo do meio (SMYTH JR, 1990).

O número de aves ornamentais criadas em cativeiro tem se elevado a cada ano, devido suas belas cores e formas e por serem consideradas animais de exposição. Assim o aumento de sua procura tem gerado preocupação com à saúde interna e externa destas aves (STERZO et al., 2012).
Diversos ectoparasitos infestam estes animais causando graves irritações locais, diminuição da postura, perda de peso, anemia e óbito. Fato este, afeta a produção e gera problemas econômicos. Dentre os ectoparasitos, os piolhos (Insecta: Phthiraptera) se distribuem no corpo destes hospedeiros em regiões ou nichos bem definidos possibilitando que estas aves tornem-se inquietas e se automutilem devido ao prurido provocado pelo caminhar destes parasitos, o que ocasiona a perda das penas e a consequente alteração térmica (FONSECA et al., 2009; SANTOS et al., 2013).

Dentre as espécies de aves, a doméstica $G$. gallus é considerada uma das que são parasitadas pela maior variedade de espécies de piolhos mastigadores (Emerson, 1956). Objetivou-se relatar a infestação por Phthiraptera mastigadores Amblycera e Ischnocera em aves da espécie G. gallus, da raça Brahma, com 
variações de coloração Dark (preto) e Light (branco) em Pendências, Rio Grande do Norte (RN), Brasil.

\section{Material e Métodos}

Foram utilizadas 20 aves G. gallus da raça Brahma, criadas em sistema extensivo, no município de Pendências, RN foram inspecionadas em dezembro de 2013, para busca de ectoparasitos. Durante inspeção observou-se sinais de infestação, dentre eles inquietação e perdas de penas (Figura 1A).

Após contenção mecânica dos animais, coletou-se Phthiraptera nas áreas corporais da cabeça, pescoço, asas, dorso e ventre. Os ectoparasitos recuperados foram armazenados em frascos com álcool a $70^{\circ} \mathrm{e}$ enviados ao Laboratório de Parasitologia Animal da Universidade Federal Rural do Semi-Árido. Posteriormente, foram clareados, por tempo médio de 10 minutos, em solução de hidróxido de potássio a $10 \%$ e montados entre lâmina e lamínula. A identificação foi feita utilizando-se chaves taxonômica de EMERSON (1956), PRICE
(1977) e TUFF (1977), auxiliada por estereomicroscopia e microscópia óptica de luz.

\section{Resultados e discussão}

Os ectoparasitos foram observados em diferentes estágios de vida. Observouse que $40 \%$ dos animais apresentaram infestação. Recuperou-se 220 Phthiraptera classificados como: 80 Lipeurus caponis, 92 Menopon gallinae e 48 Goniocotes sp.

Os piolhos da subordem Ischnocera e família Philopteridae, identificados na presente pesquisa, foram classificados por apresentar em sua morfologia antenas expostas. L. caponis apresenta têmporas arredondadas com corpo e cabeça alongados, podendo ainda apresentar mancha mediana no tórax (Figura 1B). Goniocotes sp. apresenta tamanho pequeno e coloração amarelo-pálido com cabeça circular (Figura 1C).

Classificados como Amblycera e Menoponidae, Menopon gallinae foi identificado por apresentar ausência de antenas expostas e presença de tufos de 
cerdas no $3^{\circ}$ segmento abdominal (Figura 1D, 1F).

No Brasil, parasistismo por $L$. caponis, M. gallinae e Goniocotes sp. em galinhas domésticas da espécie G. gallus foi igualmente estudado por GUERRA et al. (2008), na Paraíba; SANTOS et al. (2011), no Maranhão e por FERREIRA et al. (2012), no RN.

As descrições feitas por PINTO et al. (2001) e FONSECA et al. (2009) de que o parasitismo por piolhos em aves tornam as mesmas inquietas e diminuem a sua produção, também foram observadas na presente pesquisa. Fato este pode está associado ao número elevado destes ectoparasitos nos hospedeiros estudados.

Apesar de no RN, FONSECA et al. (2009) terem relatado infestação natural por L. caponis, M. gallinae e Goniocotes sp. em galinhas domésticas da espécie $G$. gallus, a partir dos dados da presente pesquisa, notifica-se o parasitismo por estes Phthiraptera também em G. gallus, porém para a raça Brahma, faz-se o primeiro registro neste Estado brasileiro.

\section{Conclusão}

Registra-se a infestação por $L$. caponis, M. gallinae e Goniocotes sp. em G. gallus, da raça Brahma, com variações de coloração Dark (preto) e Light (branco) em Pendências, RN, Corroborando com dados da literatura sobre estes Phthiraptera afetarem a sanidade e bem estar de aves em sistemas extensivos ou semi-intensivo, no Brasil.

\section{Referências bibliográficas}

EMERSON, K.C. Mallophaga (chewing lice) occurring on the domestic chicken. J. Aransas. Entomological Society of America, v. 29, p. 63-79, 1956.

FERREIRA， C.G.T.; BEZERRA, A.C.D.S.,; AHID, S.M.M. Inquérito Ectoparasitológico em galinhas caipiras, Gallus gallus domesticus L., do município de Apodi, Rio Grande do Norte, Brasil. Revista Brasileira de Zoociências, v.12(3), p. 1-5, 2012. 
FIGUEIREDO, E.A.P.; SCHMIDT, G.S.; LEDUR, M.C.; AVILA, V.S. Raças e linhagens de galinhas para criações comerciais e alternativas no Brasil. Concórdia: Embrapa Suínos e Aves. Comunicado Técnico, 347, 8p, 2003.

FONSECA, Z.A.A.S.; FERREIRA, C.G.T.; BEZERRA, A.C.D.S.; AHID, S.M.M. Ectofauna parasitária em aves criadas no semi-árido do Rio Grande do Norte, Brasil. Pubvet, Londrina,v.3 (10), art. 535, 2009.

GUERRA, R. M. S. N. C.; CHAVES, E.P.; PASSOS, T.M.G.; SANTOS, A.C.G. Espécies, Sítios de Localização, Dinâmica e Estrutura de Populações de malófagos em galinhas caipiras (Gallus gallus L.) criadas na ilha de São Luis, MA. Neotropical Entomology, v.37, (3), p. 259-264, 2008.

PINTO, C.; POSSATI, M.; VILLAÇA, A.; GUERIM, L.; SÁ-FREIRE，L.; SERRAFREIRE, N.M. Ocorrência de malófagos em galinhas caipiras e sua relação com o padrão de coloração da plumagem.
Entomología y Vectores, v.8(3), p.295301, 2001.

PRICE, R.D. The Menacanthus (Mallophaga: Menoponidae) of the passeriformes (Aves). Journal of Medical Entomology, v.14 (2), p.207-220, 1977. SANTOS, A.C.G.; RODRIGUES，A.L.; SANTOS, S.B.; LIMA, R.C.A.; GUERRA, R.M.S.N.C. Phthiraptera (Arthropoda, Insecta) in Gallus gallus from isolated and mixed backyard rearing systems. Revista Brasileira de Parasitologia Veterinária, v. 20, (1), p. 17-21, 2011.

SANTOS， L.S.S; SANTOS， L.M.J.F.; AGUIAR, C.L.G.; Ruas J.L.; FARIAS, N.A.R. Parasitismo de Gallus gallus (Linnaeus, 1758) por espécies de phthiraptera em criações coloniais na região sul do Rio Grande do Sul, Brasil. Arquivos do Instituto Biológico, v.80, (2), p.217-221, 2013.

SMYTH JUNIOR, J. Genetics of plumage, skin and eye pigmentation in chickens. In: CRAWFORD, R.D. Poultry breeding and 
genetics. New York: Elsevier, Cap. 5, nobre (ara maracana) - relato de caso. In: p.109-167, 1990. Congresso da Sociedade Paulista de STERZO, E.V.; GUERRA, E.J. Zoológicas - SPZOO, 2012. São Paulo, SARTORE, L.F.; PERSOLLI, L.P.; SP. Anais (on-line). Disponível: SARTORE, Y.G.A.; STEFANO,R.B.; $\quad$ http://www.spzoo.org.br/anais/publicações LAGASSI, T.C.; PACHALY, J.R.; /anais-2012. Acesso em 24 de maio de VOLTARELLI, E.M.; MONTEIRO 2014.

FILHO, L.P.C. Tratamento da síndrome de arrancamento de penas (sap) com decanoato de haloperidol em ararinha
TUFF, D.W. A key to lice of man and domestic animals. Texas Journal of Science, v.20, p.1-4, 1977. 

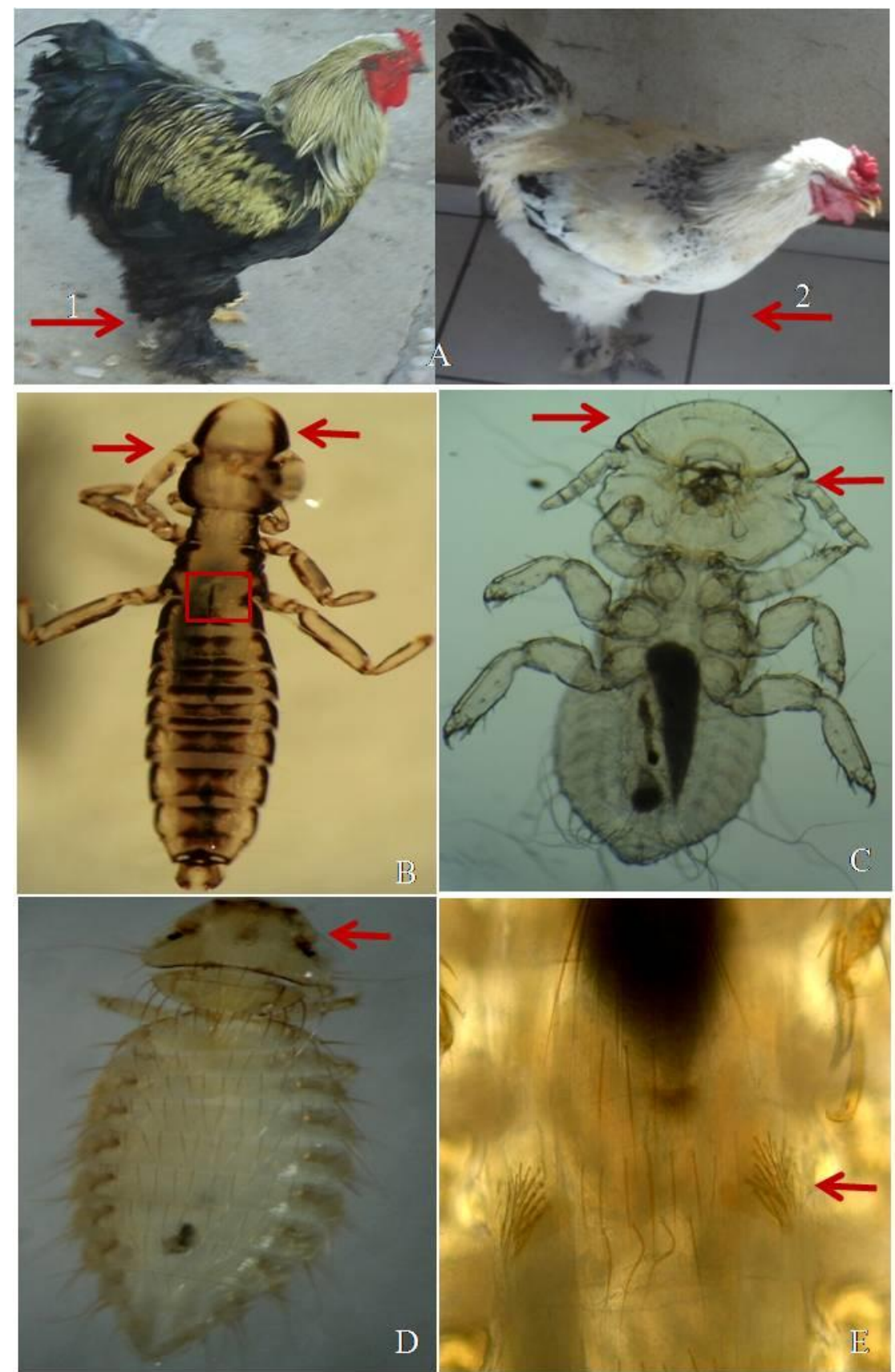

Figura 1- A- Gallus gallus da raça Brahma de coloração preto, indicado através da seta 1 e branco, através da seta 2, no município de Pendências; B- Lipeurus caponis com destaque para antenas, cabeça e mancha mediana no tórax; C-Goniocotes sp. com ênfase para cabeça e antena (objetiva 10X); Menopon gallinae: D- destaque para ausência das antenas expostas; Epresença de tufos de cerdas no $3^{\circ}$ segmento abdominal (objetiva 20X). 